'Departamento de Neurología. Facultad de Medicina. Pontificia Universidad Católica de Chile ${ }^{2}$ Hospital Base de Puerto Montt.

Servicio de Neurología Hospital Dr. Sótero del Río Santiago, Chile. ${ }^{4}$ Centro CIME Clínica los Andes, Puerto Montt.

Conflicto de interés: Los autores declaran

no tener conflictos de interés relacionados a esta publicación.

Financiamiento: Esta publicación no fue financiada por fuentes externas.

Recibido el 1 de junio de 2010 aceptado el 15 de septiembre de 2010.

Correspondencia a: Carlos Juri Clavería Departamento de Neurología. Facultad de Medicina. Pontificia Universidad Católica de Chile. Marcoleta 350. Segundo piso. Santiago, Chile. Teléfono: 56-2-3543316 E-mail: cjuri@med.puc.cl

\section{Temblor ortostático como causa de inestabilidad postural en enfermedad de Parkinson. Caso clínico}

\author{
ENRIQUE AGUILAR ${ }^{1,2,4}$, DEBORA POLLAK ${ }^{1}$, CECILIA NECOCHEA $^{1}$, \\ RICARDO FADIC ${ }^{1}$, CARLOS JURI ${ }^{1,3}$
}

\section{Orthostatic tremor causing postural instability in Parkinson disease. Report of one case}

Parkinson disease (PD) is a movement disorder characterized clinically by the variable combination of rigidity, bradykinesia, rest tremor and postural instability. Usually postural instability is a late-onset manifestation and is frequently associated with axial manifestations and with a poor prognosis. We report a 67-year-old female with orthostatic tremor as the etiology of her postural instability. The patient was treated with increasing doses of clonazepam, reaching $2 \mathrm{mg} /$ day, and levodopa. There was an improvement of postural instability with a good response of parkinsonian symptoms.

(Rev Med Chile 2010; 138: 1410-1413).

Key words: Joint instability; Parkinson disease; Tremor.
L a enfermedad de Parkinson (EP) es un trastorno del movimiento que se caracteriza clínicamente por la presencia de rigidez muscular, enlentecimiento de los movimientos (bradicinesia), temblor de predominio en reposo, trastorno de la marcha e inestabilidad postural ${ }^{1}$. Habitualmente esta última manifestación aparece tardíamente en la evolución de la enfermedad y suele asociarse a otras manifestaciones axiales y característicamente es un marcador de pobre pronóstico funcional y escasa respuesta a levodopa, en comparación con aquellos sujetos con predominio de los síntomas apendiculares ${ }^{2}$.

El temblor ortostático es una forma de temblor, generalmente de alta frecuencia, presente casi exclusivamente en la postura de pie y que afecta en mayor medida a las extremidades inferiores, manifestándose como inestabilidad postural ${ }^{3,4}$. Debido a su alta frecuencia no es visible al observador ni es percibido por el paciente y sólo es detectable al examen clínico por la palpación de las extremidades afectadas ${ }^{3}$. En los últimos años han surgido diversos reportes demostrando la presen- cia de temblor ortostático en sujetos afectados de $\mathrm{EP}^{4,5}$. A continuación presentamos el caso de una paciente con esta asociación clínica y discutimos los alcances de la misma.

\section{Caso clínico}

Mujer de 67 años, con antecedentes familiares de temblor de características esenciales en su padre y una hermana. Sin otros antecedentes mórbidos relevantes. Desde los 35 años presentaba temblor de predominio en acción en extremidades superiores, de amplitud moderada y que empeoraba en situaciones de estrés, sin repercusión funcional en sus actividades habituales. Desde hace 10 años notó aumento de intensidad del temblor, por lo que consultó. Inició tratamiento episódico con propranolol hasta $120 \mathrm{mg} /$ día y primidona hasta $250 \mathrm{mg} /$ día, con mejoría parcial. Hace 3 años se agregó temblor de reposo braquial derecho asociado a enlentecimiento de los movimientos finos ipsilaterales y cierta torpeza en la marcha, mayor 

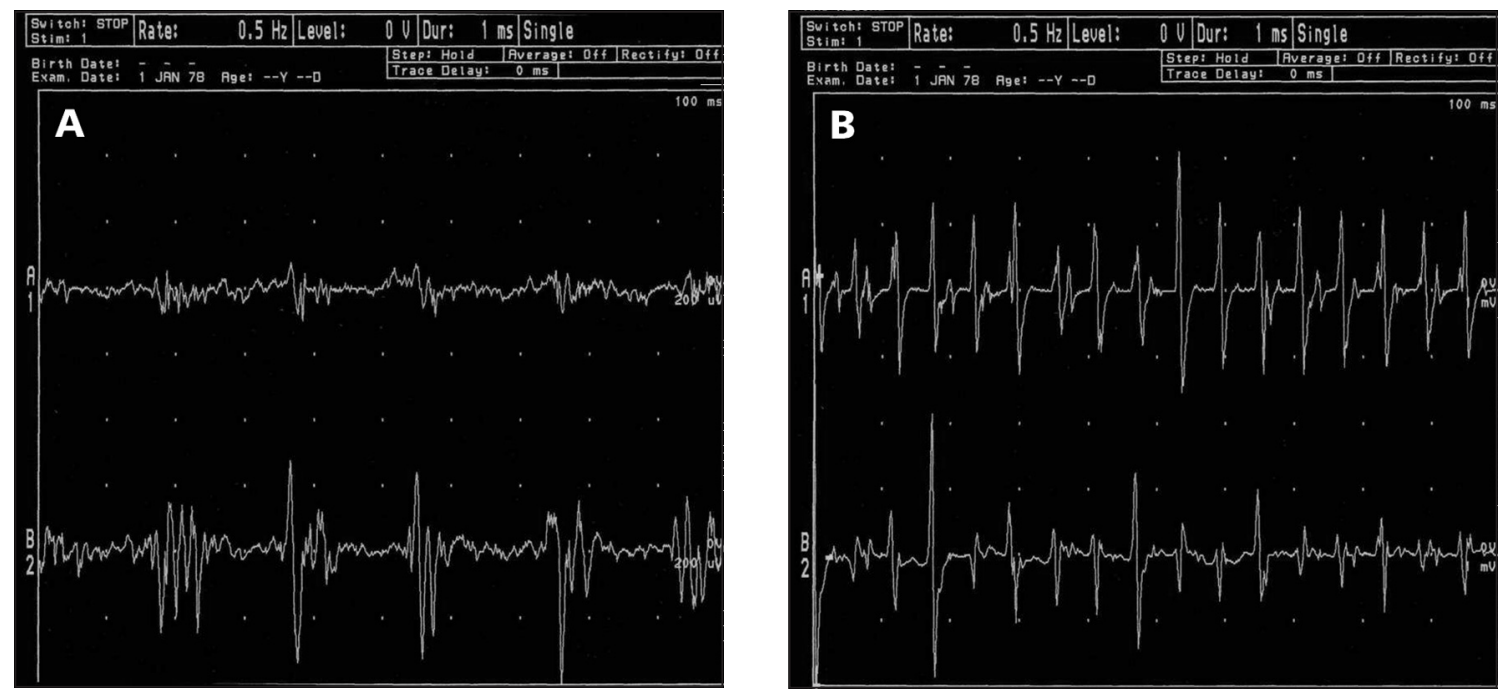

Figura 1. Registro electrofisiológico con electrodos de superficie sobre el músculo vasto lateral derecho. En (A) se observa el registro de temblor de baja frecuencia $(4 \mathrm{a} 6 \mathrm{~Hz}$ ) en decúbito dorsal. En (B) se aprecia el registro de temblor de alta frecuencia $(16 \mathrm{~Hz})$ en posición de pié, con las características de temblor ortostático. Velocidad de registro $10 \mathrm{~cm} / \mathrm{seg}$.

con la pierna derecha. Por este motivo inició tratamiento con levodopa/carbidopa en dosis progresivas hasta 250/25 medio comprimido 3 veces al día, con mejoría significativa del temblor de reposo y disminución de la bradicinesia. Desde hace 2 años presenta inestabilidad postural, especialmente en la bipedestación mantenida, que suele aliviar marcadamente al caminar o al sentarse. Por este síntoma se indicó aumento de la dosis de levodopa/carbidopa (250/25), hasta 3 comprimidos al día, sin notar mejoría en la estabilidad postural. En su evolución no refería fluctuaciones motoras, como tampoco discinesias asociadas al uso de la medicación dopaminérgica.

Al momento de nuestra evaluación la paciente se encontraba sin consumo de levodopa desde hacía 12 horas. $\mathrm{Al}$ examen destacaba la presencia de rigidez muscular de moderada intensidad en extremidades derechas con presencia de Froment a izquierda, también presentaba enlentecimiento leve de los movimientos finos a derecha, sin bradicinesia evidente a izquierda. Además existía temblor de reposo de baja frecuencia braquial derecho y crural ipsilateral y temblor de acción braquial bilateral de moderada frecuencia y baja amplitud. En la posición de pie destacaba una importante inestabilidad postural, que aliviaba marcadamente al caminar. No se observaba temblor en las piernas en la posición de pie, sin embargo, era posible palpar una actividad rítmica en ambos muslos, más intensa a derecha en dicha postura. En la situación motora "ON", bajo efecto de levodopa, se apreció mejoría de la rigidez, bradicinesia y del temblor de reposo, sin embargo, la inestabilidad postural se mantuvo. La prueba de retropulsión forzada ("pull-test") era normal. Llamaba la atención la ausencia de manifestaciones axiales destacables, había mínima hipomimia sin hipofonía. El examen de la presión arterial era normal, sin evidencias de ortostatismo.

Clínicamente se interpretó como portadora de temblor esencial familiar de larga data, asociado a un cuadro parkinsoniano de predominio derecho de 3 años de evolución, con buena respuesta a levodopa y con inestabilidad postural asociada a actividad muscular rítmica de alta frecuencia en extremidades inferiores, sugerente de temblor ortostático. Frente a esta última opción se solicitó estudio electrofisiológico con el objetivo de descartar la existencia de temblor ortostático.

El estudio electrofisiológico realizado con electrodos de superficie demostró un temblor de reposo de 4 a 6 Hertz (ciclos por segundo) en extremidad superior e inferior derecha. En la postura de pie se evidencia un cambio de frecuencia a 16 $\mathrm{Hz}$ del temblor en las extremidades inferiores, sin variaciones en el temblor de extremidades superiores. Al retomar la posición decúbito el temblor en las piernas volvía a la frecuencia de 4 a $6 \mathrm{~Hz}$. inicial (Figura 1). 
Con estos elementos se planteó el diagnóstico de temblor ortostático secundario asociado a síndrome parkinsoniano, probablemente EP idiopática. Iniciamos clonazepam en dosis crecientes hasta $2 \mathrm{mg}$ al día, manteniendo la dosis de levodopa, con lo que hubo mejoría de la inestabilidad postural y mantuvo la buena respuesta de los síntomas parkinsonianos.

\section{Comentario}

La EP es un trastornos neurodegenerativo que se caracteriza por la muerte predominante de neuronas dopaminérgicas de la sustancia nigra pars compacta $^{6}$. Este daño genera diversas manifestaciones motoras y no motoras ${ }^{7}$. En particular, da cuenta de la presencia de rigidez y bradicinesia propias de la EP. Sin embargo, otras manifestaciones como el temblor no han sido claramente explicadas en humanos ni en modelos experimentales por esta afectación. Se ha planteado que la presencia de temblor en EP se relacionaría con el déficit serotoninérgico o de otras vías de neurotransmisión ${ }^{8}$.

El temblor ortostático es una condición que aparece en la edad media de la vida y suele presentarse como sensación de inestabilidad asociado a marcha temerosa, sin signología cerebelosa y con escasas caídas ${ }^{3}$. Su causa es desconocida y presenta una asociación poco clara con temblor esencial. Se desconoce qué sistemas de neurotransmisión o regiones cerebrales son las más afectadas ${ }^{9}$. Tanto la frecuencia como la amplitud del temblor ortostático no son influenciadas por estímulos nerviosos periféricos, sugiriendo un origen central ${ }^{10}$. Esto es apoyado por estudios que han usado estimulación magnética transcraneana sobre la corteza motora, provocando una modulación del temblor ortostático medido en extremidades inferiores. Una posible explicación plantea la existencia de dos circuitos, uno intrínseco dado por conexiones estriato-tálamo-córtico-estriatales, y un circuito extrínseco-tálamo cortical, encargado de estabilizar las oscilaciones del circuito intrínseco y que sería modulado por la estimulación cerebral transcreanana ${ }^{11}$. La participación subcortical también ha sido sugerida en estudios con PET que muestran activación cerebelosa anormal bilateral, así como de tálamo y lenticular contra-lateral en un individuo que padecía de temblor ortostático en bipedestación ${ }^{12}$. La participación de la vía ves- tibular en este cuadro también ha sido investigada, sin embargo, no se ha reportado modulación del temblor con cambios de posición de la cabeza.

El temblor ortostático puede ser aliviado completamente al levantar al individuo del piso y se reproduce en extremidades superiores al apoyarse en las 4 extremidades sobre el piso. El estudio de la marcha muestra que el temblor desaparece en la extremidad que se levanta y se mantiene en la pierna de soporte. Estos elementos apuntarían al papel fundamental que ejerce la contracción isométrica de las extremidades para la manifestación del temblor, en teoría de origen central ${ }^{13}$. A pesar de todos estos indicios no es posible explicar la sensación de inestabilidad que se alivia al deambular tan típicamente descrito por los pacientes.

Ocasionalmente se ha descrito temblor en ortostatismo en pacientes con EP, aunque generalmente de baja frecuencia, similar al temblor de reposo $(4-6 \mathrm{~Hz})$ y con buena mejoría con levodopa en estos casos. La presencia de temblor ortostático de alta frecuencia $(14-16 \mathrm{~Hz})$ en el contexto de pacientes con EP plantea la existencia de interacción entre ambas entidades ${ }^{5}$, lo que se apoya en la presencia de respuesta parcial a agentes dopaminérgicos y el empeoramiento del temblor ortostático asociado a la progresión del parkinsonismo.

Casos anecdóticos también han descrito la aparición e temblor ortostático en el contexto de déficits nutricionales de tiamina, planteándose como una posibilidad diagnóstica a investigar frente a la aparición de esta manifestación ${ }^{14}$.

Recientemente se ha clasificado el temblor ortostático asociado a EP en 3 grupos: lento ( 4 a $6 \mathrm{~Hz})$, intermedio $(7 \mathrm{a} 9,5 \mathrm{~Hz})$ y rápido $(10$ a 18 $\mathrm{Hz}$ ), además del mioclonus ortostático ${ }^{4}$. El más frecuentemente descrito es el rápido, como en nuestra paciente, que además remeda el temblor ortostático primario (no asociado a otras enfermedades). Este tipo de temblor rápido tendría un origen independiente de la inervación dopaminérgica estriatal como ha sido demostrado por la ausencia de denervación dopaminérgica en estudios de radioligandos ${ }^{15} \mathrm{y}$ en los casos con denervación dopaminérgica ella sigue un patrón distinta de la observada en la $\mathrm{EP}^{15,16}$. El temblor ortostático rápido tiene pobre mejoría con levodopa y agonistas dopaminérgicos ${ }^{17,18}$, siendo su repuesta preferentemente al uso de clonazepam ${ }^{4,19}$.

El caso presentado enfatiza la importancia de 
evaluar con detención la presencia de inestabilidad postural en portadores de EP, dado que la detección de temblor ortostático en estos pacientes determina cambios en el pronóstico funcional y en el tratamiento. Este caso también demuestra la utilidad de realizar un estudio electrofisiológico en pacientes con sospecha clínica de temblor ortostático $^{20}$.

\section{Referencias}

1. Tolosa E, Wenning G, Poewe W. The diagnosis of Parkinson's disease. Lancet Neurol 2006; 5: 75-86.

2. Poewe W. The natural history of Parkinson's disease. Journal of Neurology 2006; 253: 2-6.

3. Piboolnurak P, Yu QP, Pullman SL. Clinical and neurophysiologic spectrum of orthostatic tremor: case series of 26 subjects. Mov Disord 2005; 20: 1455-61.

4. Leu-Semenescu S, Roze E, Vidailhet M, Legrand AP, Trocello JM, Cochen V, et al. Myoclonus or tremor in orthostatism: An under-recognized cause of unsteadiness in Parkinson's disease. Mov Disord 2007; 22: 2063-9.

5. Apartis E, Tison F, Arne P, Jedynak CP, Vidailhet M. Fast orthostatic tremor in Parkinson's disease mimicking primary orthostatic tremor. Mov Disord 2001; 16: 1133-6.

6. Braak H, Del Tredici K, Rub U, de Vos RA, Jansen Steur EN, Braak E. Staging of brain pathology related to sporadic Parkinson's disease. Neurobiol Aging 2003; 24: 197-211.

7. Borek LL, Amick MM, Friedman JH. Non-motor aspects of Parkinson's disease. CNS Spectrums 2006; 11: 541-54.

8. Wolters EC, Braak H. Parkinson's disease: premotor clinico-pathological correlations. J Neural Transm Suppl 2006; 70: 309-19.

9. Jedynak P, Agid Y. Primary orthostatic tremor and unsteadiness. Mov Disord 2004; 19: 989.

10. Britton TC, Thompson PD, van der Kamp W, Rothwell
JC, Day BL, Findley LJ, et al. Primary orthostatic tremor: further observations in six cases. J Neurol 1992; 239: 209-17.

11. Tsai CH, Semmler JG, Kimber TE, Thickbroom G, Stell $\mathrm{R}$, Mastaglia FL, et al. Modulation of primary orthostatic tremor by magnetic stimulation over the motor cortex. J Neurol Neurosurg Psychiatry 1998; 64: 33-6.

12. Wills AJ, Thompson PD, Findley LJ, Brooks DJ. A positron emission tomography study of primary orthostatic tremor. Neurology 1996; 46: 747-52.

13. Boroojerdi B, Ferbert A, Foltys H, Kosinski CM, Noth J, Schwarz M. Evidence for a non-orthostatic origin of orthostatic tremor. J Neurol Neurosurg Psychiatry 1999; 66: 284-8.

14. Nasrallah KM, Mitsias PD. Orthostatic tremor due to thiamine deficiency. Mov Disord 2007; 22: 440-1.

15. Vaamonde J, García A, Flores JM, Ibáñez R, Gargallo L. Study of presynaptic nigrostriatal pathway by $123-\mathrm{I}-\mathrm{FD}$ CIT-SPECT (DatSCAN SPECT) in primary orthostatic tremor. Neurología 2006; 21:37-9.

16. Katzenschlager R, Costa D, Gerschlager W, O'Sullivan J, Zijlmans J, Gacinovic S, et al. 123I]-FP-CIT-SPECT demonstrates dopaminergic deficit in orthostatic tremor. Ann Neurol 2003; 53: 489-96.

17. Wu YR, Ashby P, Lang AE. Orthostatic tremor arises from an oscillator in the posterior fossa. Mov Disord 2001; 16: 272-9.

18. Wills AJ, Brusa L, Wang HC, Brown P, Marsden CD. Levodopa may improve orthostatic tremor: case report and trial of treatment. J Neurol Neurosurg Psychiatry 1999; 66: 681-4.

19. Gerschlager W, Munchau A, Katzenschlager R, Brown P, Rothwell JC, Quinn N, et al. Natural history and syndromic associations of orthostatic tremor: a review of 41 patients. Mov Disord 2004; 19: 788-95.

20. Glass GA, Ahlskog JE, Matsumoto JY. Orthostatic myoclonus: a contributor to gait decline in selected elderly. Neurology 2007; 68: 1826-30. 